MscI, a type II restriction endonuclease from Micrococcus species which recognizes 5' TGGCCA $3^{\prime}$

\title{
Carol Polisson
}

New England Biolabs, 32 Tozer Road, Beverly, MA 01915, USA

Submitted June 14, 1989

Msc I, a type II restriction endonuclease, has been isolated from Micrococcus species ( NEB $\# 502$ ). Msc I, an isoschizomer of $B a l$ I (1), recognizes the palindromic hexanucleotide sequence 5' TGGCCA 3'. Like Ball, Msc I cleaves the sequence, TGG/CCA, generating a blunt end.

The single cleavage site of Msc I on M13mp18 DNA was mapped to approximately position 5095 by analysis against Bgll, Bsm I and Dra III cleaved M13mp18 DNA (figure I, lanes 2-5). The sequence 5' TGGCCA 3' occurs at position 5080. The number and sizes of the fragments generated by digestion with Msc I on eight DNA molecules ( 39 sites) match the computer predicted (2) number and sizes of the fragments that would be generated by cleavage at the sequence 5'TGGCCA3'. Msc I has the following number of recognition sites on these commonly used DNAs: pUC19 (0), pBR322 (1), phiX174 (0), SV40 (0), M13mp18 (1), T7 (2), Lambda (18) and Adeno2 (17), (figure I, lanes 6-8). From these data we conclude that Msd recognizes the sequence 5' TGGCCA 3'. The crude extract contains approximately 1000 units $\mathrm{Mscl}$ per gram of cells.

The cleavage site of Msct was determined by cleavage of a primed synthesis reaction (2). Using a DNA fragment known to contain the sequence 5'TGGCCA3' as template with an appropriate primer, the four standard dideoxy sequencing reactions were performed and a fifth reaction was extended through the Msc I site. The fifth reaction was terminated by heat treatment. Mscl was added to the fifth reaction. The cleaved product resulted in a single band ( figure II, lane - ) comigrating with a band in the G channel of the sequencing reactions. The addition of Klenow fragment subsequent to $M s c I$ digestion resulted in a single band comigrating with the same G residue ( fig. II, lane +). These results indicate that $M s c \mathrm{I}$, exactly like its isoschizomer Bal l, cleaves after the second Guanine nucleotide within the recognition sequence in the $5^{\prime}$ to $3^{\prime}$ direction on both strands producing a blunt end.

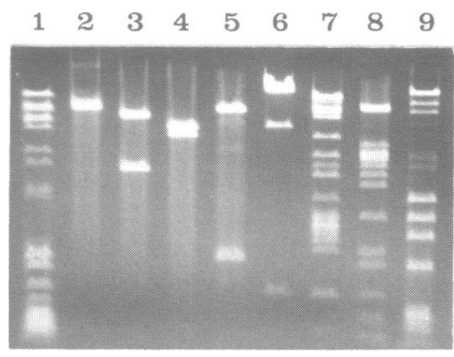

Figure I. Msc I digests of DNA. Lane 2, uncut M13mp18; lane 3, M13mp18 $+M s c l$ + Bgl II; lane 4, M13mp18 + Msc I + Bsml; lane 5, M13mp18 + Msc I + Dra III, Lanes 6-8: T7, Lambda and Adeno2, respectively. Markers: lane 1, BstEll-Lambda and Msp I-pBR322; lane 5, Hind IIILambda and Hae III-phiX174.

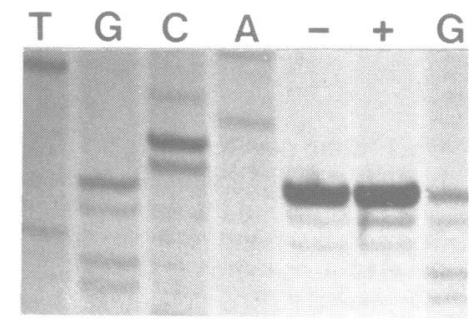

Figure II.

\section{REFERENCES}

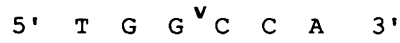

$$
\begin{aligned}
& \text { 3. } A \text { C } C_{\Lambda} G \text { G T } 5^{\prime}
\end{aligned}
$$

1. Gelinas R.E., Myers P.A., Weiss G.A., Roberts R.J., and Murray K.E. (1977) J. Mol. Biol. 114, 433-440.

2. Devereaux J., Haeberli P., and Smithies O. (1984) NAR 12, 387-395.

3. Brown N.L., Hutchison C.A., and Smith M. (1980) J. Mol. Biol. 140, 143-148. 